\title{
Dynamical behavior of liquid in reservoir of revolution under harmonic force disturbance in the below resonant frequency range
}

\author{
Limarchenko O., Parankina O., Slyusarchuk Yu. \\ The Taras Shevchenko KNU \\ Glushkov avenue, 4-e, Kiev, Ukraine
}

(Received 26 December 2017)

\begin{abstract}
Dynamical behavior of liquid in a reservoir of revolution under vibration disturbance of reservoir motion is studied within the framework of the model of combined motion. We focus main attention on the system behavior in below resonant range. For analysis of peculiarities of the system behavior we compare the development of wave generation for cylindrical, conic, spherical, hyperboloid (one-sheet and two-sheet) reservoirs.
\end{abstract}

Keywords: reservoir, vibrations, combined motion, free-surfaced liquid, non-cylindrical shape, drift of oscillations, modulation, high-frequency modes.

2000 MSC: 76B07, 76B99

UDC: 532.595

DOI: $10.23939 / \mathrm{mmc} 2017.02 .156$

\section{Introduction}

Structures containing reservoirs with a free-surfaced liquid are used in aerospace engineering, for liquid storage and transport. The problem of development of the model of combined motion of structures with liquid in the case of non-cylindrical shape of reservoirs is urgent. Analysis of the system behavior under force loading shows that new types of dynamic effects manifests for combined modes of motion. The ratio of masses of liquid and reservoir, peculiar for combined motion [1], considerably affects the development of liquid and reservoir motions and even values of normal frequencies of oscillations. Recent investigations $[2,3]$ showed that system behavior for below resonant, near resonant and above resonant ranges differ considerably. In the present article we focus our attention of the above resonant range of oscillations for horizontal motion of the reservoir under harmonic force, applied to the reservoir. The cases of cylindrical, conic, spherical, hyperboloid (one-sheet and two-sheet) reservoirs are considered for comparison. The problem is studied within the framework of nonlinear combined model of motion with considering 10 normal modes of oscillations.

Objective of the present publication is study of peculiarities of the system behavior in the below resonant range of force disturbance and analysis of effect of reservoir shape on development of wave processes.

\section{Mathematical model}

Known publication [1-8] considered nonlinear problems of oscillations of liquid in reservoirs of cylindrical and conic shapes. We investigate problems of waves generation for other shapes of the reservoirs in combined mode of motion. For investigation of such types of the problems we make use of the mathematical method [4], developed for arbitrary type of reservoir of revolution.

Let us denote $\tau$ to be the domain, occupied by liquid; $S_{0}$ and $S$ are a liquid free surface in its perturbed and unperturbed motion; $\Sigma_{0}$ and $\Sigma$ are boundaries of the contact of liquid with reservoirs walls in perturbed and unperturbed motion; $\eta(x, y, z, t)=0$ is the equation of a liquid free surface; $\varepsilon$ is the vector of displacements of the reservoir with respect to absolute reference frame. Liquid is supposed to be ideal, homogeneous, incompressible and initially its motion is vortex-free. Effect of 
capillary forces is supposed to be negligible. The reservoir is considered as absolute rigid body. Under these assumptions, the liquid motion can be described by the velocity potential.

Mathematical statement of the problem about motion of the system reservoir-liquid - consists of kinematical constraints, dynamical conditions, dynamical equations and initial conditions. Kinematical conditions are considered as mechanical constraints, which should be satisfied before application of the variational principle of Hamilton-Ostrogradsky. For the considered system kinematical conditions include the continuity requirement in the domain $\tau$

$$
\Delta \varphi=0 \quad \text { in } \quad \tau
$$

nonflowing condition on the boundary of contact reservoir-liquid $\Sigma$ with the unit vector of external normal $n$

$$
\frac{\partial \varphi}{\partial n}=\dot{\boldsymbol{\varepsilon}} \cdot \boldsymbol{n} \quad \text { on } \quad \Sigma
$$

and nonflowing condition on a free surface of liquid

$$
\frac{\partial \xi}{\partial t}+\nabla \xi \cdot \nabla \varphi=\frac{\partial \varphi}{\partial z} \quad \text { on } \quad S
$$

The dynamical boundary condition corresponds to the requirement of pressure balance on a free surface of liquid

$$
\frac{\partial \varphi}{\partial t}+\frac{1}{2}(\nabla \varphi)^{2}-\nabla \varphi \cdot \dot{\varepsilon}-\boldsymbol{g} \cdot \boldsymbol{r}=0 \quad \text { on } \quad S .
$$

The velocity potential for liquid is represented as $\Phi=\varphi+\dot{\varepsilon} \cdot \boldsymbol{r}$, where $\boldsymbol{r}$ is the radius-vector of points of the domain $\tau$. The first term corresponds to wave motion of liquid, the second one is connected with translational motion of the reservoir.

According to [4], the dynamical boundary conditions and the motion equations are obtained from the Hamilton-Ostrogradsky variational principle

$$
\delta \int_{t_{1}}^{t_{2}} L d t=0
$$

with the Lagrange function

$$
L=\frac{1}{2} \rho \int_{\tau}(\nabla \varphi)^{2} d \tau+\frac{1}{2} M_{p}(\dot{\varepsilon})^{2}-\frac{1}{2} \rho g \int_{S_{0}} \xi^{2} d S-\left(M_{p}+M_{f}\right) \varepsilon_{z} g
$$

where $\rho$ is the liquid density, $M_{p}$ is the reservoir mass, $M_{f}$ is the liquid mass, $g$ is the free falling acceleration.

For passing to the resolving system of ordinary differential equations, we use decomposition of unknown variables into series according to [4], and take into account that for vortex-free motion of homogenous incompressible ideal liquid motion of liquid boundary completely characterizes motion of its volume

$$
\xi=\xi(t)+\sum_{i} a_{i} \bar{\psi}_{i}(\alpha) T_{i}(\theta)
$$

After elimination of kinematical conditions according to the method [4], we obtain the variational problem for a free system for which it is possible to write the Lagrange equations of the second kind

$$
\sum_{i} \ddot{a}_{i}\left\{V_{i r}^{1}+\sum_{j} a_{j} V_{i r j}^{2}+\sum_{j, k} a_{j} a_{k} V_{i r j k}^{3}\right\}+\ddot{\varepsilon} \cdot\left\{\boldsymbol{U}_{r}^{1}+\sum_{i} a_{i} \boldsymbol{U}_{r i}^{2}+\sum_{i, j} a_{i} a_{j} \boldsymbol{U}_{r i j}^{3}+\sum_{i, j, k} a_{i} a_{j} a_{k} \boldsymbol{U}_{r i j k}^{4}\right\}
$$




$$
\begin{array}{r}
=\sum_{i, j} \dot{a}_{i} \dot{a}_{j} V_{i j r}^{2 *}+\sum_{i, j, k} \dot{a}_{i} \dot{a}_{j} a_{k} V_{i j k r}^{3 *}+\dot{\varepsilon}\left\{\sum_{i} \dot{a}_{i} \boldsymbol{U}_{i r}^{2 *}+\sum_{i, j} \dot{a}_{i} a_{j} \boldsymbol{U}_{i r j}^{3 *}+\sum_{i, j, k} \dot{a}_{i} a_{j} a_{k} \boldsymbol{U}_{i j k r}^{4 *}\right\} \\
-g\left\{\sum_{i} a_{i} W_{i r}^{2}+\frac{3}{2} \sum_{i, j} a_{i} a_{j} W_{i j r}^{3}+2 \sum_{i, j, k} a_{i} a_{j} a_{k} W_{i j k r}^{4}\right\}, \quad r=1,2, \ldots, N \\
\frac{\rho}{\left(M_{p}+M_{f}\right)}\left\{\sum_{i} \ddot{a}_{i}\left[\boldsymbol{U}_{i}^{1}+\sum_{j} a_{j} \boldsymbol{U}_{i j}^{2}+\sum_{j} a_{j} a_{k} \boldsymbol{U}_{i j k}^{3}\right]\right\}+\ddot{\boldsymbol{\varepsilon}} \\
=\frac{F}{\left(M_{p}+M_{f}\right)}-g \boldsymbol{z}_{0}-\frac{\rho}{\left(M_{p}+M_{f}\right)} \sum_{j} \dot{a}_{j} \dot{a}_{j}\left\{\boldsymbol{U}_{i j}^{2}+2 \sum_{k} a_{k} \boldsymbol{U}_{i j k}^{3}\right\} .
\end{array}
$$

The construction of the nonlinear discrete model of dynamics of combined motion of the reservoir with liquid was done based on the Kantorovich method applied to the variational statement of the problem. Here two generalized coordinates were used, namely, $a_{i}$ are amplitudes of excitations of the normal modes of liquid oscillations; $\varepsilon_{i}$ are parameters of translational motion of the reservoir.

In the general form we represent the motion equations for the system as

$$
\sum_{n=1}^{N} p_{r n}\left(a_{k}, t\right) \ddot{a}_{n}+\sum_{n=N+1}^{N+3} p_{r n}\left(a_{k}, t\right) \ddot{\varepsilon}_{n-N}=q_{r}\left(a_{k}, \dot{a}_{l}, t\right), \quad r=\overline{1, N+3} .
$$

Here $p_{r n}$ is a quadratic matrix, $q_{r}$ is a column of dimension $N$. The expressions for $p_{r n}$ and $q_{r}$ can be recovered from the system (1), (2).

\section{Numerical results}

Let us consider combined motion of the reservoir with liquid of radius $R$ in the horizontal plane under the action of the external periodic force $F=A \sin (\omega t)$. Here $H$ is liquid filling level.

The problem is studied for the frequencies $0.5 \omega_{N}$ and $0.75 \omega_{N}$, where $\omega_{N}$ is the frequency of normal oscillations of combined motion of liquid with the reservoir, which depends on ratio of their masses and does not equal the partial frequency of oscillations of liquid in an immovable reservoir. For translational motion of the reservoir this difference can exceed 50-70\%. Amplitudes of force were selected such that maximum amplitudes of oscillations of a free surface were within the range $0.15 R-0.2 R$.

System behavior was analyzed for time interval about $50 \mathrm{~s}$, which corresponds to about 40 periods of oscillations. It was assumed that the reservoir mass is equal to 0.2 of the liquid mass. For the considered reservoirs we take $R=1 \mathrm{~m}$, the liquid depth is $H=R$ for all reservoirs besides the spherical one, where $H=0.5 R$. We consider the resolving system, which takes into account 10 normal modes of oscillations. Previously, this model was successfully tested for fulfillment of conservation laws $[4,8]$. At the same time, the results for a conic reservoir qualitatively coincide with the results of experimental publications $[6,8]$.

Simultaneously with investigation of behavior of liquid in the below resonant frequency range we are going to investigate peculiarities of the influence of geometric shape on the development of dynamical processes. We consider and compare numerical results for cylindrical, conic, spherical and hyperbolical (one-sheet and two-sheet) reservoirs. Let us analyze amplitudes of excitation of liquid sloshing on tank walls in time for the frequencies of force disturbance $0.5 \omega_{N}$ and $0.75 \omega_{N}$. Figs. $1-8$ represent the dependence of amplitudes on time. The parameter $A$ is the amplitude of exciting force. Figs. 1-3 correspond to the frequency $0.5 \omega_{N}$; Figs. $4-6$ correspond to the frequency $0.75 \omega_{N}$. For the first frequency range drift of mean value of amplitudes of oscillations manifests. At the same time the presence of high frequency modes of oscillations in the resulting amplitude of oscillations is considerable. 
The general tendency of behavior of amplitude - time dependence for different shapes of reservoirs is similar; however, manifestation of both drift of mean value of oscillations and the presence of highfrequency modes is stronger for the conic reservoir. In the case of oscillations with exciting frequency $0.75 \omega_{N}$ (Figs. 4-6) the presence of modulation dominates, and similar to the previous frequency range for the conic reservoir this effect manifests stronger. Similar results are represented for one-sheet and two-sheet hyperboloid in Figs. 7-10 for $0.5 \omega_{N}$ (Figs. 7, 8) and $0.75 \omega_{N}$ (Figs. 9-10). General behavior of the system does not change, however, it is necessary to note that manifestation of high-frequency modes for one-sheet hyperboloid is much stronger than for other reservoirs, besides the conic one.

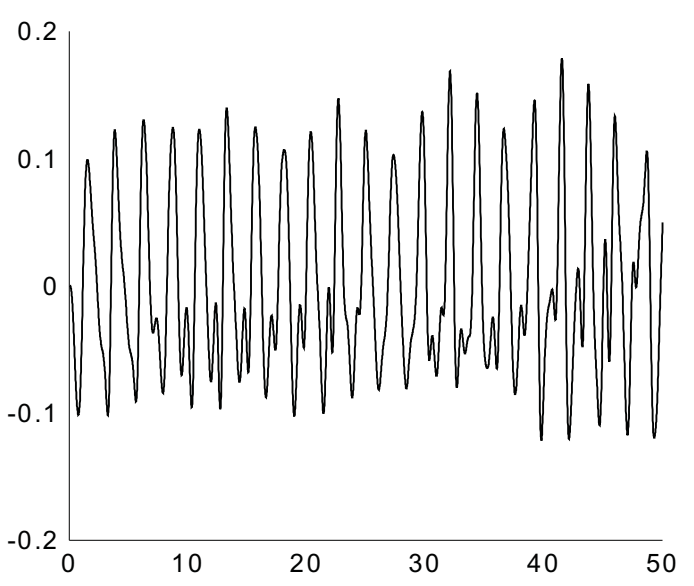

Fig. 1. Amplitude-time dependence for cylinder $A=0.7$.

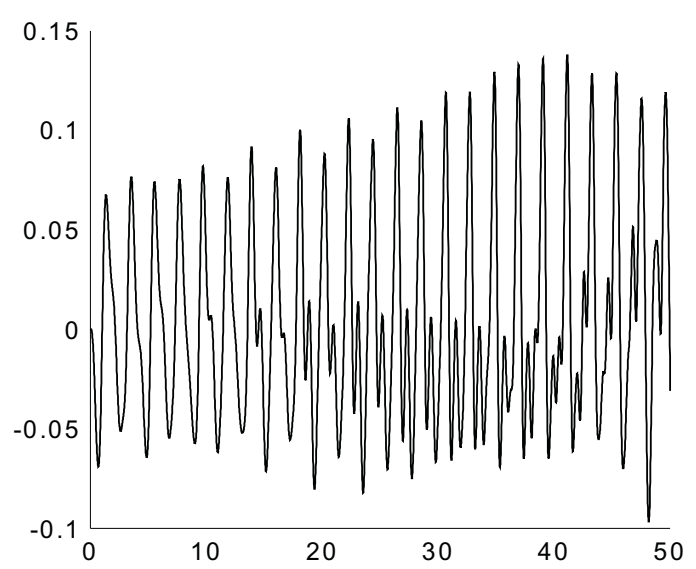

Fig. 3. Amplitude-time dependence for sphere $A=0.5$.

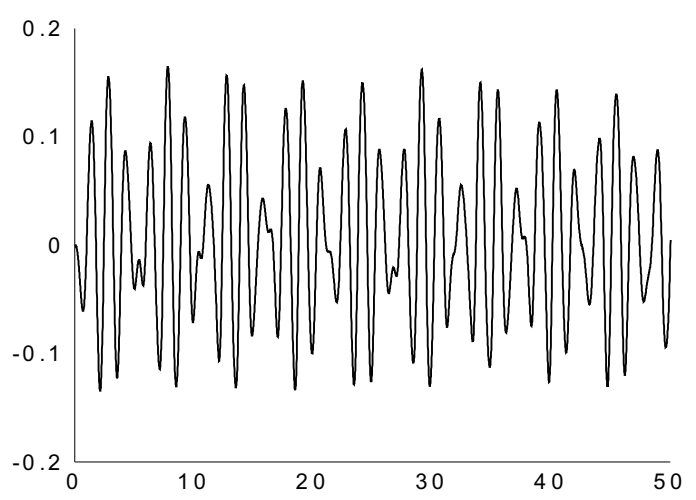

Fig. 5. Amplitude-time dependence for cone $A=0.35$.

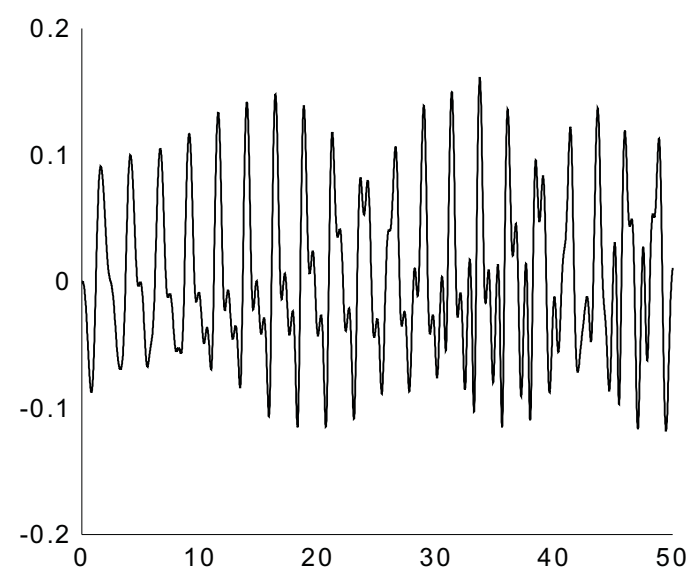

Fig. 2. Amplitude-time dependence for cone $A=0.5$.

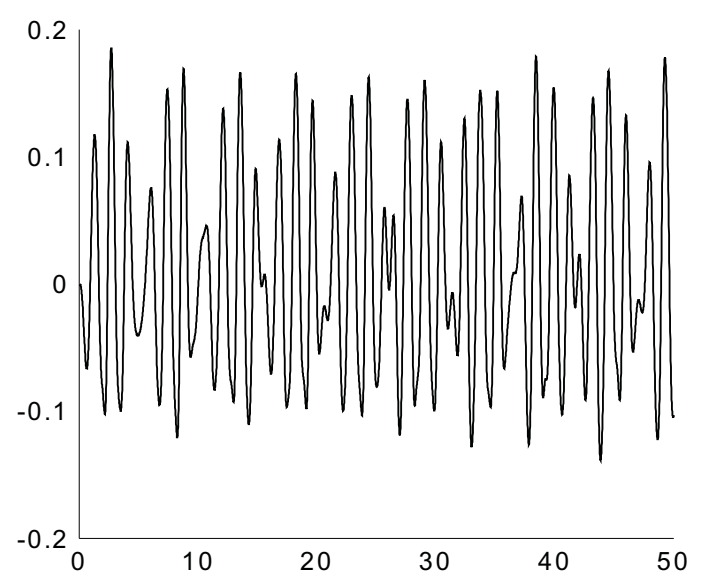

Fig. 4. Amplitude-time dependence for cylinder $A=0.4$.

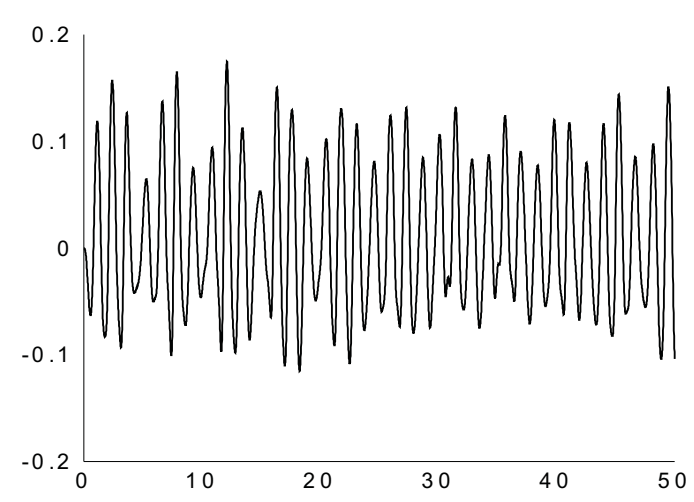

Fig. 6. Amplitude-time dependence for sphere $A=0.45$.

Mathematical Modeling and Computing, Vol.4, No. 2, pp. 156-161 (2017) 


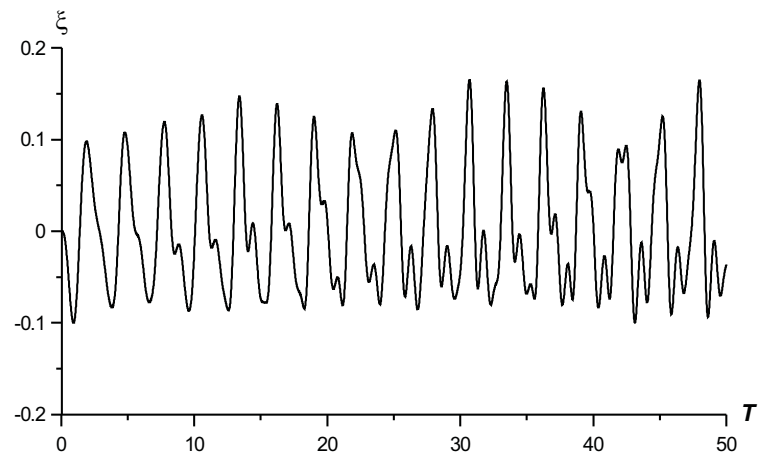

Fig. 7. Amplitude-time dependence for one-sheet hyperboloid $A=0.42$.

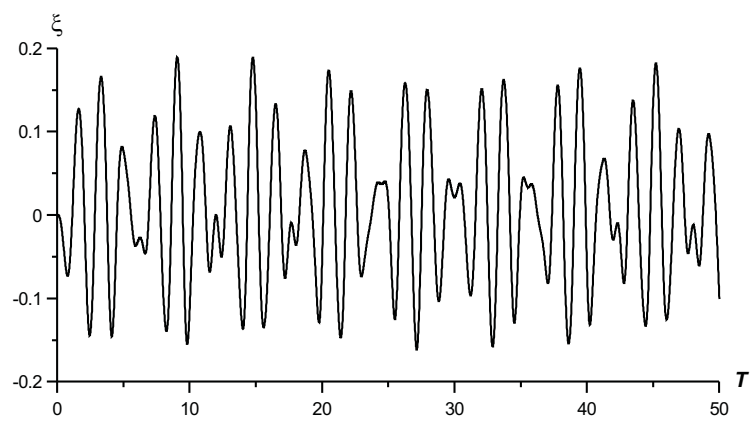

Fig. 9. Amplitude-time dependence for for onesheet hyperboloid $A=0.3$.

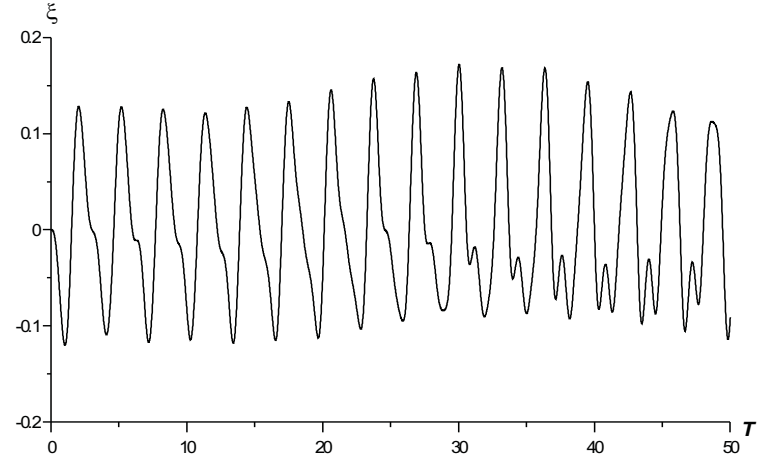

Fig. 8. Amplitude-time dependence for two-sheet hyperboloid $A=0.4$.

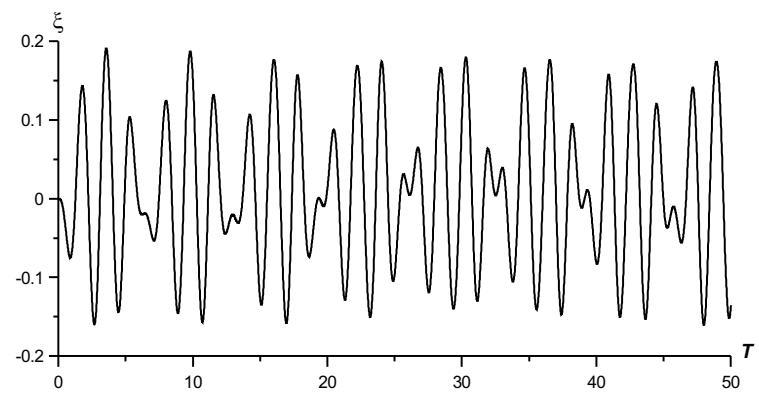

Fig. 10. Amplitude-time dependence for twosheet hyperboloid $A=0.25$.

The general property for all cases of reservoirs shapes and for both ranges of frequencies is that the system does not tend to steady mode of motion. This property is confirmed by the results of experiments $[6,8]$. Mathematically this is caused by the simultaneous considering of both exciting frequencies (including their multiple values) and normal frequencies of all modes of oscillations, which are not divisible. In contrast to this, the result publications $[5,6]$ neglect the mentioned normal frequencies of oscillations and obtain results of system asymptotical tending to a steady mode of oscillations, which is not confirmed experimentally. One of the attribute of the necessity of inclusion of normal modes of oscillations with their eigenfrequencies is reflected in the strong manifestation of high-frequency modes in variation of amplitudes of liquid motion on the reservoir wall. It is necessary to note also that on increase of perturbation frequency contribution of high-frequency modes decreases and the effect of modulation of oscillations dominates.

\section{Conclusions}

The problem of force motion of liquid with a free surface in movable reservoir of revolution was considered. Numerical results were obtained for cylindrical, conic, spherical and hyperbolic (one-sheet and two-sheet) reservoirs. We analyzed variation in time of excitations on tank walls of a free surface for the frequency range smaller that the resonant frequency of normal oscillations (below frequency range).

It was ascertained that for the below frequency range drift of means value of oscillations is strongly manifested. On tending of the perturbing frequency to the resonant value effect of modulation of 
oscillations dominates. Manifestation of high-frequency modes occurs too. The effect of geometrical shape of reservoir on these properties was discussed also.

For all shapes of reservoirs and different perturbing frequencies the system has no tendency of converging to a steady mode of motion, which is confirmed experimentally $[6,8]$.

[1] Konstantinov O., Limarchenko O., Semenovich K. Effect of combine motion on variation of resonance properties in liquid sloshing problems. Math. Model. Comput. 2 (1), 48-57 (2015).

[2] Limarchenko O. S., Matarazzo G., Yasinsky V. V. Dynamics of rotating structures with liquid. Kiev, Gnosis (2002).

[3] Limarchenko O. S., Gubskaya V.V. Problem of forced nonlinear oscillations of the reservoir of truncated conic shape, partially filled with liquid. Bulletin of Kiev National University. 1 (4), 73-76 (2012).

[4] Limarchenko O. S., Yasinsky V. V. Nonlinear dynamics of structures with liquid. Kiev, NTUU "KPI" (1997).

[5] Narimanov G.S., Dokuchayev L. V., Likovskiy I. A. Nonlinear dynamics of flying objects with liquid. Moscow, Mashinostroyeniye (1977).

[6] Faltinsen O. M., Rognebakke O.F., Timokha A.N. Transient and steady-state amplitudes of resonant three dimensional sloshing in a square base tank with a finite fluid depth. Physics of fluids. 18 (1), 012103-1012103-14 (2006).

[7] Limarchenko O. S., Semenova I. Yu. Nonlinear wave generation on a fluid in a moving parabolic tank. International Applied Mechanics. 46 (8), 864-868 (2011).

[8] Pal P. Sloshing of liquid in partially filled container - an experimental study. International Journal of Recent Trends in Engineering. 1(6), 1-5 (2009).

\title{
Динамічна поведінка резервуара в формі тіла обертання під дією гармонічної сили в дорезонансному діапазоні частот
}

\author{
Лимарченко О., Паранкіна О., Слюсарчук Ю. \\ Київсъкий начіональний університет імені Тараса Шевченка \\ пр. Глушкова, 4-е, Київ, Украӥна
}

\begin{abstract}
Вивчено динамічну поведінку резервуара в формі тіла обертання під дією гармонічної сили в межах моделі сумісного руху. Головну увагу зосереджено на поведінці системи в дорезонансному діапазоні зміни частот. Для аналізу особливостей поведінки системи порівняно розвиток хвилеутворення для резервуарів циліндричної, конічної, сферичної і гіперболоїдальної (однопопорожнинний і двопорожнинний) форм.
\end{abstract}

Ключові слова: резервуар, вібрацї̈, з'єднаний рух, вільна поверхня рідина, нециліндрична форма, дрейф коливань, модуляціл, високочастотні моди.

2000 MSC: 76B07, 76B99

УдК: 532.595 